Article

\title{
Gene Regulation Network Analysis on Human Prostate Orthografts Highlights a Potential Role for the JMJD6 Regulon in Clinical Prostate Cancer
}

\author{
Mario Cangiano ${ }^{1}$ (D), Magda Grudniewska ${ }^{1}$ (D), Mark J. Salji ${ }^{2,3}$, Matti Nykter ${ }^{4}$, Guido Jenster ${ }^{5}$ (D), \\ Alfonso Urbanucci ${ }^{6} \mathbb{D}^{\mathbb{D}}$, Zoraide Granchi ${ }^{1}{ }^{\mathbb{D}}$, Bart Janssen ${ }^{1}$, Graham Hamilton ${ }^{7} \mathbb{D}$, Hing Y. Leung ${ }^{2,3, * \mathbb{D}}$ \\ and Inès J. Beumer $1, *$
}

Citation: Cangiano, M.; Grudniewska, M.; Salji, M.J.; Nykter, M.; Jenster, G.; Urbanucci, A.;

Granchi, Z.; Janssen, B.; Hamilton, G.; Leung, H.Y.; et al. Gene Regulation Network Analysis on Human Prostate Orthografts Highlights a Potential Role for the JMJD6 Regulon in Clinical Prostate Cancer. Cancers 2021, 13, 2094. https://doi.org/ 10.3390/cancers13092094

Academic Editor: Michael W. Krainer

Received: 1 March 2021

Accepted: 21 April 2021

Published: 26 April 2021

Publisher's Note: MDPI stays neutral with regard to jurisdictional claims in published maps and institutional affiliations.

Copyright: (c) 2021 by the authors. Licensee MDPI, Basel, Switzerland. This article is an open access article distributed under the terms and conditions of the Creative Commons Attribution (CC BY) license (https:/ / creativecommons.org/licenses/by/ $4.0 /)$.
1 GenomeScan B.V. Plesmanlaan 1D, 2333 BZ Leiden, The Netherlands; 2352539C@student.gla.ac.uk (M.C.); m.grudniewska@genomescan.nl (M.G.); zoraide.granchi@gmail.com (Z.G.); b.janssen@genomescan.nl (B.J.)

Institute of Cancer Sciences, University of Glasgow, Glasgow G61 1QH, UK; Mark.Salji@ggc.scot.nhs.uk CRUK Beatson Institute, Glasgow G61 1BD, UK

4 Laboratory of Computational Biology, Institute of Biomedical Technology, Arvo Ylpön katu 34, 33520 Tampere, Finland; matti.nykter@uta.fi

5 Department of Urology, Erasmus Medical Center, Doctor Molewaterplein 40, 3015 GD Rotterdam, The Netherlands; g.jenster@erasmusmc.nl

6 Department of Tumor Biology, Institute for Cancer Research, Oslo University Hospital, 0424 Oslo, Norway; alfonsou@ifi.uio.no

7 Glasgow Polyomics, University of Glasgow, Glasgow G61 1QH, UK; Graham.Hamilton@glasgow.ac.uk

* Correspondence: h.leung@beatson.gla.ac.uk (H.Y.L.); i.beumer@genomescan.nl (I.J.B.)

Simple Summary: Prostate cancer is a very common malignancy worldwide. Treatment resistant prostate cancer poses a big challenge to clinicians and is the second most common cause of premature death in men with cancer. Gene expression analysis has been performed on clinical tumours but to date none of the gene expression-based biomarkers for prostate cancer have been successfully integrated to into clinical practice to improve patient management and treatment choice. We applied a novel laboratory prostate cancer model to mimic clinical hormone responsive and resistant prostate cancer and tested whether a network of genes similarly regulated by transcription factors (gene products that control the expression of target genes) are associated with patient outcome. We identified regulons (networks of genes similarly regulated) from our preclinical prostate cancer models and further evaluated the top ranked JMJD6 gene related regulated network in three independent clinical patient cohorts.

Abstract: Background: Prostate cancer (PCa) is the second most common tumour diagnosed in men. Tumoral heterogeneity in PCa creates a significant challenge to develop robust prognostic markers and novel targets for therapy. An analysis of gene regulatory networks (GRNs) in PCa may provide insight into progressive PCa. Herein, we exploited a graph-based enrichment score to integrate data from GRNs identified in preclinical prostate orthografts and differentially expressed genes in clinical resected PCa. We identified active regulons (transcriptional regulators and their targeted genes) associated with PCa recurrence following radical prostatectomy. Methods: The expression of known transcription factors and co-factors was analysed in a panel of prostate orthografts $(n=18)$. We searched for genes (as part of individual GRNs) predicted to be regulated by the highest number of transcriptional factors. Using differentially expressed gene analysis (on a per sample basis) coupled with gene graph enrichment analysis, we identified candidate genes and associated GRNs in PCa within the UTA cohort, with the most enriched regulon being JMJD6, which was further validated in two additional cohorts, namely EMC and ICGC cohorts. Cox regression analysis was performed to evaluate the association of the JMJD6 regulon activity with disease-free survival time in the three clinical cohorts as well as compared to three published prognostic gene signatures (TMCC11, BROMO-10 and HYPOXIA-28). Results: 1308 regulons were correlated to transcriptomic data from the three clinical prostatectomy cohorts. The JMJD6 regulon was identified as the top enriched regulon in the UTA cohort and again validated in the EMC cohort as the top-ranking regulon. In 
both UTA and EMC cohorts, the JMJD6 regulon was significantly associated with cancer recurrence. Active JMJD6 regulon also correlated with disease recurrence in the ICGC cohort. Furthermore, Kaplan-Meier analysis confirmed shorter time to recurrence in patients with active JMJD6 regulon for all three clinical cohorts (UTA, EMC and ICGC), which was not the case for three published prognostic gene signatures (TMCC11, BROMO-10 and HYPOXIA-28). In multivariate analysis, the JMJD6 regulon status significantly predicted disease recurrence in the UTA and EMC, but not ICGC datasets, while none of the three published signatures significantly prognosticate for cancer recurrence. Conclusions: We have characterised gene regulatory networks from preclinical prostate orthografts and applied transcriptomic data from three clinical cohorts to evaluate the prognostic potential of the JMJD6 regulon.

Keywords: prostate cancer; prognostic biomarkers; gene regulatory network; regulon; transcriptional regulator

\section{Background}

Prostate cancer (PCa) is the second most common cancer among men and the fifth leading cause of death worldwide [1]. Tumour heterogeneity in PCa (between patients and among different tumour foci within individual patients) creates a major obstacle to the identification of clinically relevant molecular subtypes [2]. As a result, PCa treatment decisions are not based on tumour biology. Disease recurrence following treatment remains a significant problem, even following radical treatment such as radical prostatectomy or radical radiotherapy [3]. Despite the use of docetaxel chemotherapy or second generation androgen receptor pathway inhibitors along with androgen deprivation therapy (ADT), patients presenting with advanced and/or metastatic disease are at high risk of recurrent disease, which tend to be aggressive and incurable as either castration resistant (CRPC) or neuroendocrine PCa variants $[4,5]$. Therefore, there is an unmet need to improve our understanding of progressive PCa in order to identify new targets for therapy as well as prognostic biomarkers.

Inter-patient tumoral heterogeneity and intra-tumour heterogeneity among different tumour foci are well reported, making it unlikely that a single gene will be a representative biomarker of PCa progression [6]. Investigating a gene set-related network may leverage the correlations of the expression of multiple interacting genes [7]. Several gene set-based panels are offered as prognostic tests for PCa patients. Commercial assays [8-10] including Decipher ${ }^{\mathrm{TM}}$, Oncotype DX ${ }^{\circledR}$ and Prolaris, together with scoring methods published in the literature, have been developed using microarray, Illumina or Nanostring transcriptome profiles [9-11] to apply mRNA expression data to predict the risk of cancer recurrence and/or progression. While gene expression-based models have resulted in promising data for predicting cancer behaviour in vitro [11], significant improvements are required before a stratification/prognostic tool in PCa patients can be considered for routine clinical practice, including the prediction of the risk of cancer recurrence following treatment [12]. The limitations of existing commercial molecular PCa diagnostic tests may stem from potential biases introduced during the signature identification step (including factors related to patient ethnicity [13], immune [14] and stromal [15] components of the tumours) that may influence the gene expression profiles. Moreover, gene set-based methods typically focus on the expression of individual genes or gene sets, without the ability to incorporate biologically important information associated with gene-gene interactions [7].

Alterations in transcriptional programmes are frequently implicated in PCa progression [16]. Genes that co-operate within the same biological pathways are often under the regulatory control of shared (one or more) transcription factors. Conveniently, interacting genes tend to be associated at the expression levels [17], providing the chance to infer their relationships from transcriptomics data. Gene regulatory networks (GRNs) are graphs describing transcriptional regulators and their target genes as nodes, while the relationships 
(level of correlation) among the regulators and target genes are presented as the edges. Statistical and/or machine learning approaches have been applied to gene expression data [18] to predict the topology of GRNs, namely the arrangement of transcriptional regulators and their target genes as well as the direction of each transcription factor-target interaction (i.e., positive or negative regulation). Within GRNs, data on the agreement between the predicted regulations and differential gene expression analysis can be applied to explore the underlying biological mechanisms to explain specific phenotypes (such as cancers with lower or higher chances of recurrence/progression).

Preclinical models of human PCa cells grown as orthotopic xenografts in mice (orthografts) represent a useful tool to mimic progressive clinical disease. However, the use of preclinical PCa as a tool to identify potential GRNs involved in progressive disease has not been tested. Here, to generate a robust scoring method, we derived GRNs from a collection of preclinical hormone naïve (dependent on androgens for growth) and castration resistant (growth despite androgen deprivation therapy) human PCa orthografts to capture the heterogeneous nature of clinical disease, leveraging the strength of correlations in the expression patterns of genes transcribed by tumour cells only. Filtering the GRNs for statistically significant associations led to the identification of putative regulons, signifying the network of target genes and shared transcription factor (or transcriptional regulator) involved. Integrating data from preclinical orthografts and clinical PCa cohorts, we modelled regulon signatures to identify patients at risk of cancer recurrence, and identified the JMJD6 (Jumonji Domain Containing 6, arginine demethylase and lysine hydroxylase, a protein hydroxylase or histone demethylase) regulon as a prognostic marker in PCa (Figure 1).



Figure 1. Workflow summarising the study analysis pipeline.

\section{Materials and Methods}

See Supplementary Information for additional details for datasets and methods.

\subsection{Datasets}

Hormone naïve human prostate cancer cell lines (CWR, LNCaP and VCaP) were implanted into the prostates of androgen proficient (6 weeks old) nude male mice to generate androgen dependent prostate orthografts. Castration resistant (or androgen independent) prostate orthografts were generated from the 22Rv1, LNCaPAI and VCaPR 
human PCa cell lines by orthotopic implantation into the prostates of castrated nude ( 6 weeks old) male mice. RNA-seq data were obtained from 18 orthografts derived from the six human PCa cell lines studied ( $n=3$ mice per cell line) [19], referred to as the UGLA dataset. All data were included for the inference of the gene regulatory network.

RNA-seq data from three clinical PCa cohorts were included in this study: The University of Tampere (UTA-EGAD00001000609), the Erasmus Medical Center in Rotterdam (EMC-EGAD00001004215), and the International Cancer Genome Consortium (ICGCEGAD00001004791). A summary of the clinicopathological characteristics of the cohorts is provided in Table 1.

Table 1. Clinicopathological characteristics of patient cohorts (NA, data not available).

\begin{tabular}{|c|c|c|c|c|c|c|}
\hline Clinical Cohorts & UTA & & EMC & & ICGC & \\
\hline Number $(n)$ & $n=27$ & $\%$ & $n=37$ & $\%$ & $n=85$ & $\%$ \\
\hline \multicolumn{7}{|l|}{ age at diagnosis } \\
\hline range & $47-71$ & & NA & & $32-52$ & \\
\hline mean & 60 & & NA & & 47 & \\
\hline median & 61 & & NA & & 48 & \\
\hline na & 0 & & & & & \\
\hline \multicolumn{7}{|l|}{ psa at diagnosis (ng/mL) } \\
\hline range & $3.5-48.1$ & & $0.3-36.2$ & & $3.1-743$ & \\
\hline mean & 10.4 & & 11.8 & & 30.48 & \\
\hline median & 8.3 & & 9.4 & & 8.21 & \\
\hline na & 0 & & 0 & & 0 & \\
\hline \multicolumn{7}{|l|}{ tumour stage } \\
\hline $\mathrm{t} 1$ & 10 & 37.0 & 1 & 2.7 & 0 & 0.0 \\
\hline $\mathrm{t} 2$ & 16 & 59.3 & 15 & 40.5 & 61 & 71.8 \\
\hline $\mathrm{t} 3$ & 1 & 3.7 & 13 & 35.1 & 23 & 27.1 \\
\hline $\mathrm{t} 4$ & 0 & 0.0 & 8 & 21.6 & 1 & 1.2 \\
\hline na & 0 & 0.0 & 0 & 0.0 & 0 & 0.0 \\
\hline \multicolumn{7}{|l|}{ gleason score } \\
\hline$<7$ & 7 & 25.9 & 6 & 16.2 & 12 & 14.1 \\
\hline 7 & 13 & 48.2 & 19 & 51.4 & 65 & 76.5 \\
\hline$>7$ & 7 & 25.9 & 0 & 0.0 & 8 & 9.4 \\
\hline na & 0 & 0.0 & 12 & 32.4 & 0 & 0.0 \\
\hline \multicolumn{7}{|l|}{ therapy } \\
\hline Radical prostatectomy & 27 & 100 & 37 & 100 & 85 & 100 \\
\hline
\end{tabular}

Dataset from the UTA cohort [20] were obtained from 46 prostate tumour samples, including 28 untreated PCa samples from radical prostatectomy and 12 benign prostate hyperplasia control samples (obtained by radical prostatectomy, cystoprostatectomy or transurethral resection). RNA-seq data from treatment naive PCa samples that passed mapping quality control, provided with information on progression free time $(n=27)$, were used in this study, along with the 12 benign samples.

The EMC dataset was obtained from 92 radical prostatectomy specimens (51 PCa with 41 adjacent benign prostate tissue) [21,22]. The tumour content was confirmed histologically. Only prostate tumour samples with the information on progression free time $(n=37)$ and all the benign control samples were used in the present study. 
The ICGC dataset consists of 125 PCa specimens (and 8 matched benign control tissue) from 100 radical prostatectomy specimens [23]. Six tumour samples from the same prostatectomy specimens were sampled multiple times (from 3 to 6 biological replicates per patient) and were averaged at gene count level per patient, given the similarity in expression profiles. Samples from patients that did not receive neo-adjuvant therapy $(n=85)$ and all the benign samples $(n=8)$ were used in the present study.

\subsection{Regulons Identification and Filtering}

The PCa gene-regulatory network was generated using the R package 'RTN' [24] version v2.4.6, based on FPKM values (Fragments Per Kilobase of transcript per Million mapped reads) of the UGLA orthograft dataset and a list of 2065 transcription factors that were given as input (manually curated from MsigDb [25]). Out of the 2065 transcription factors, statistically significant associations with one or more target genes were found for 1643 regulators. The normalised counts matrix was then filtered by genes with FPKM equal or higher than one in at least one sample and standardised within the zero-to-one range. The function 'tna.shadow' from the R package 'Viper' [26] version 1.14.0 has been used to account for the 'shadow' effect (the chance of obtaining false positive result) during the enrichment of a GRN, if a non-active regulator shares a significant proportion of its targets with a bona fide active transcription factor, providing a final set of 1308 regulons.

\subsection{Gene Regulatory Network Metrics}

The graph structure was analysed using the R package 'igraph' v1.2.5, exploiting the functions 'degree', 'betweenness', 'constraint' and 'closeness' to retrieve metrics at the 'nodes' level, providing complementary information about the importance of individual nodes within the network: (1) The 'degree' (or 'in-degree') of a node in a GRN is the number of transcriptional regulators involved in the control of the expression of a specific target gene. For different GRNs, the number of regulatory genes implicated for individual target genes varies, depending on complexity of the network; (2) 'Betweenness' is defined as the number of shortest paths passing through the node and can be interpreted as a measure of the influence of the node of interest over the global flow of information; (3) Burt's 'constraint' is a measure of the redundancy of the information received by the node and can be interpreted as its ability to converge different signals; (4) 'Closeness' quantifies the node's participation within a network. Finally, the Jaccard Index, a statistical measure defined as the ratio of the intersection and the union of two sets, was applied to highlight network nodes sharing a meaningful proportion of targets. The threshold of 0.1 was chosen to prioritise the nodes to be shown in Figure 2. A threshold of Jaccard Index/Co-efficient set at 0.1 highlights pairs of regulons with intersection (sharing) of $\geq 10 \%$ of the target genes when considered across the full set of target genes for the respective regulons. 


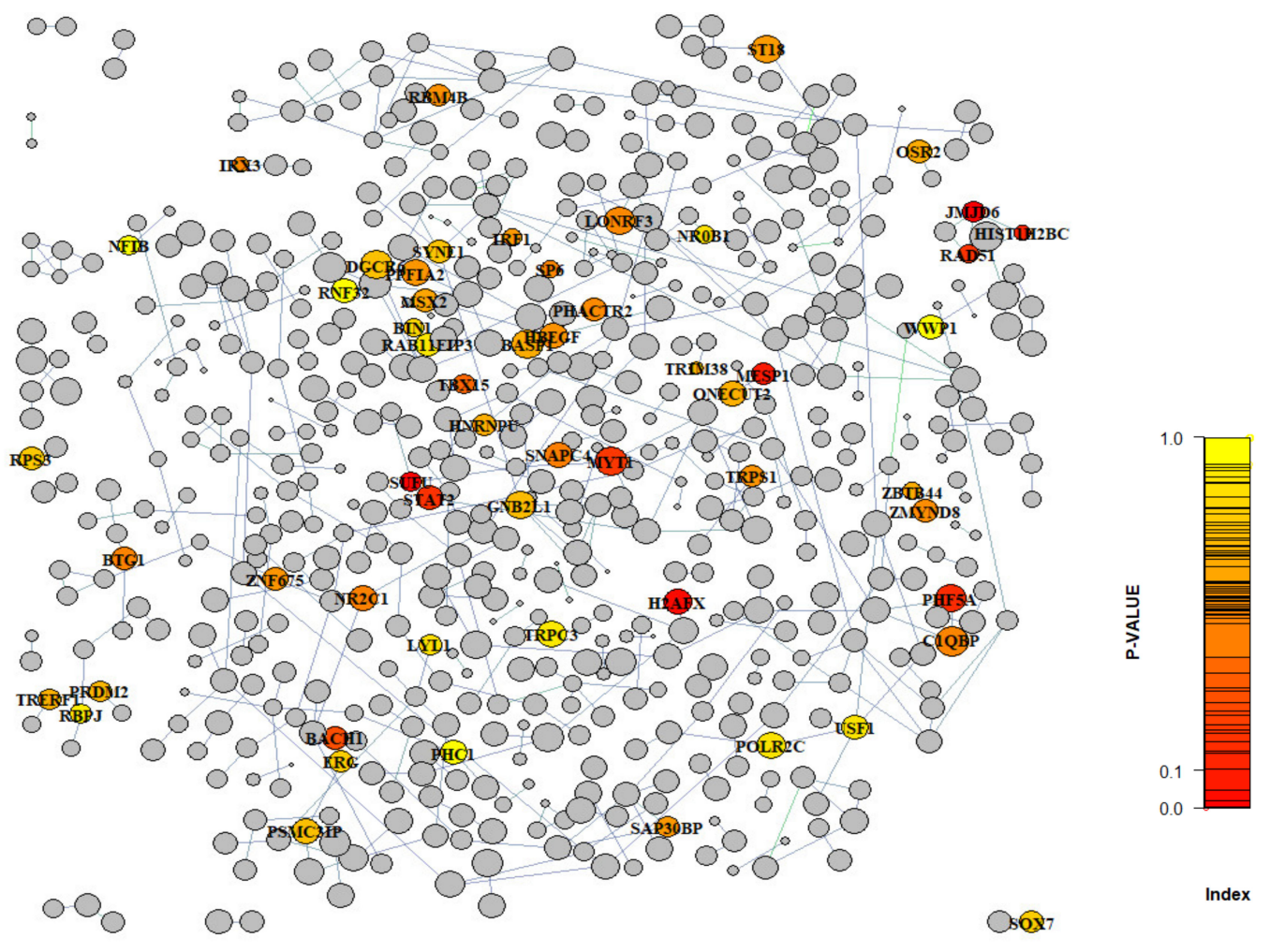

(A)

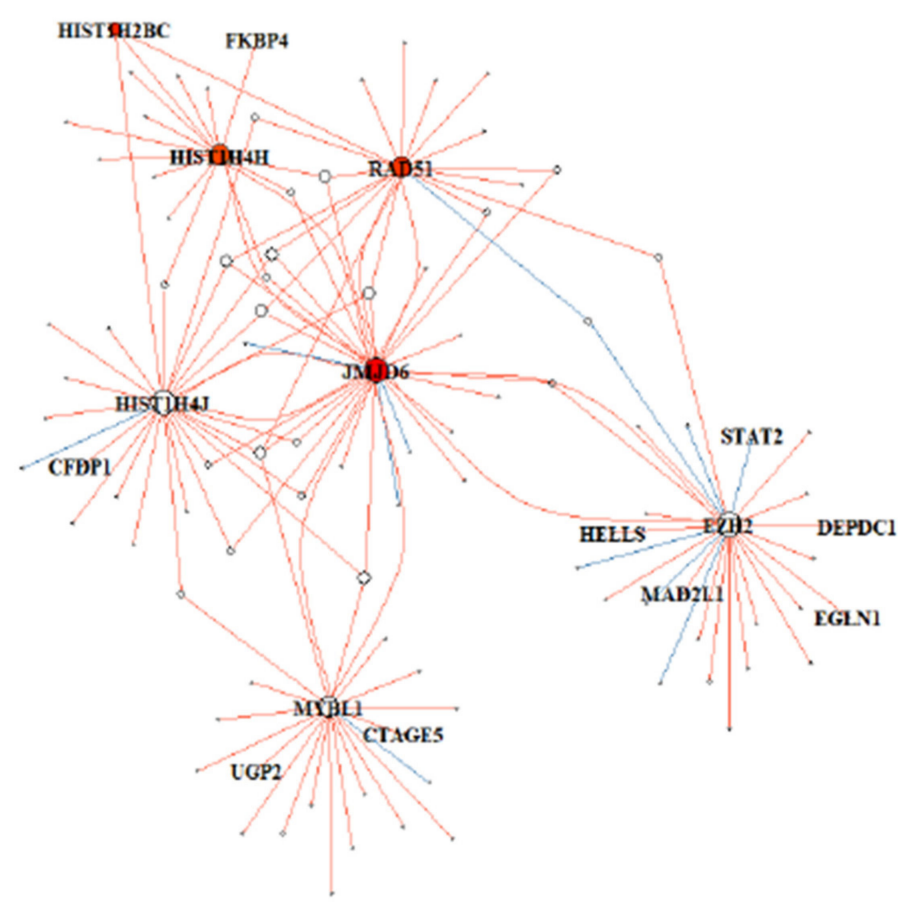

(B)

Figure 2. (A) Gene regulatory networks identified in preclinical human prostate cancer orthografts. The regulatory network of regulons (nodes of all colours) is presented with the edges linking pair of regulons sharing part of their targets. The commonality between pairs of regulons was calculated through the Jaccard index. Pairs with Jaccard Index $\geq 0.1$ are shown. 
The colour of the nodes refers to the colour scale (range 1-0) represents the $p$-value of the enriched regulons associated with relapse free survival in the clinical (UTA and EMC) cohorts by cox regression analysis. Regulons in grey represent insignificant networks and therefore not included in further analysis. (B) The gene regulatory network topology cantered on the JMJD6 regulon. Red edges represent positive regulations while blue edges inhibitory relationships. (A,B) The names of regulators are annotated with HUGO gene symbol in black. The colour scale (range 1-0) represents the $p$-value of the enriched regulons associated with disease free survival.

\subsection{Regulons Enrichment}

The list of differentially expressed genes (DEG; $\geq \log 2$ fold changes and false discovery rate, FDR, $\leq 0.05)$ for each sample was determined using the respective benign control samples within each of the clinical UTA, EMC and ICGC cohorts. $p$-values were adjusted using the function 'p.adjust ( $)$ ' from the $\mathrm{R}$ stat package v4.0.3, by setting the 'method' parameter to ' $\mathrm{fdr}$ '. The DEG gene set for each cohort was analysed using the gene regulatory network that was identified in the preclinical orthografts. The derived list of positive and negative gene-gene interactions was then used as input to the function 'nbea' from the package 'EnrichmentBrowser' [27] version v2.12.1, applying the 'GGEA' (gene graph enrichment analysis) method with default parameters. A threshold of FDR $\leq 0.05$ has been adopted to identify the enriched regulons.

\subsection{Statistical Analyses}

Biochemical recurrence was defined as serum prostate-specific antigen (PSA) levels $\geq 2 \mathrm{ng} / \mathrm{mL}$ above nadir PSA (the lowest PSA level after treatment) and signifies clinical evidence of relapsed cancer. Relapse free survival (defined by absence of biochemical recurrence) was used to evaluate the prognostic utility of regulon signatures of interest in the UTA, EMC and ICGC clinical cohorts. The performance of our candidate JMJD6 regulon signature as a prognostic marker was compared to three published signatures [28-30]. The performance of our candidate JMJD6 regulon signature as a prognostic marker was compared to three published signatures (using the formulas used in the original publications [28-30]: (1) For the TMCC11 signature, the per-sample average of the normalised counts of the genes belonging to the signature was used to stratify the patient cohort into two groups according to values above or below the 67th percentile. (2) For the HYPOXIA-28 signature, the normalised counts were multiplied by the coefficient associated to each gene of the signature and all the products were added together to generate a sample-specific overall score, and the patient cohort was stratified into two groups according to the median of its distribution. (3) For the BROMO-10 signature, the function 'gsva' from GSVA v1.38.2 was used to analyse data from the normalised counts to calculate a signature enrichment score per sample.

Patients were labelled according to the enrichment status of $J M J D 6$, as predicted by GGEA, into active or inactive status groups. Hazard ratios (HR) for all the analysis were obtained by Cox proportional-hazard model regressions, using the 'coxph' function from the R package 'survival' version 3.1-8. Moreover, for multivariate analysis, Gleason score and the TNM (Tumour/Node/Metastasis) classification were added to the model formula in the form: 'Endpoint JMJD6regulon_activity + second_variable'. Kaplan-Meier curves were obtained using the 'ggsurvplot' function from the R package 'survminer' v0.4.8. The analysis was performed in $\mathrm{R} v 4.03$.

\section{Results}

\subsection{Gene Regulatory Network Inferred from Preclinical Prostate Orthograft Models}

The expression profiles of 2064 manually curated transcription factors and cofactors [25] (Table S1) were correlated with the differentially expressed genes in 18 prostate orthografts derived from human PCa cells, namely CWR22Res, 22Rv1, LNCaP, LNCaP-AI and $\mathrm{VCaP}(n=3$, except for $\mathrm{VCaP})$. $\mathrm{VCaP}$ derived orthografts were grown in both hormone proficient and castrated mice ( $n=3$ each). 1308 regulons with a median of 20 genes per reg- 
ulon (range 2-121) were identified (Table S2). Interestingly, a large fraction of transcription factors $(n=607 ; 46.4 \%)$, shared at least one target gene (Figure $2 \mathrm{~A}$ ).

Genes controlled by multiple transcription factors at the transcriptional level may suggest a functional requirement in controlling the expression of these target genes, thus signifying the likelihood of their biological importance. We searched for genes (as part of individual GRNs) predicted to be regulated by the highest number of transcriptional factors (Table S3). Up to 10 transcription factors per target gene were observed within the networks identified. Four target genes were associated with the highest number of transcription factors $(n=10)$, and interestingly all of these four genes have previously been implicated in PCa: BUD31 encodes for a bona-fide AR-coactivator that enhances AR transactivation in prostate cells [31]; PLOD3 is involved in tissue remodelling and plays a role in multiple tumour types including PCa [32]; SDR42E1 is implicated in early prostate organogenesis as well as carcinogenesis [33] and XAGE1A belongs to the cancer testis antigens family and its expression profile is linked to the aggressiveness of PCa [34]. Hence, a GRN-based analysis of prostate orthografts generated a network of candidate transcriptional regulators and their target genes that can be evaluated in clinical tumours.

\subsection{Analysis of Differentially Expressed Genes (DEG) in Clinical PCa Patient Cohorts}

Through comparison of each clinical tumour with the combined benign controls within the respective clinical cohorts, lists of differentially expressed genes (on a per sample basis) were generated on a per-sample basis initially in the UTA clinical cohort as part of a discovery analysis. The list of PCa associated genes ( $\log 2$ fold changes and $p$-values) was then be used to identify the GRNs of interest, highlighting potential active regulons in individual tumours. In the UTA cohort ( $n=27 \mathrm{PCa})$, we found a median of 2406 upregulated (range 1098-6419) and 282 downregulated (range 44-1173) genes per sample. In the EMC cohort as a validation dataset $(n=37 \mathrm{PCa})$, we observed a median of 2439 upregulated (range 827-7395) and 126 downregulated (range 1-925) genes for individual tumour samples.

We ranked the differentially expressed genes by the average frequency of alteration (up- or down- regulation) within the respective patient cohorts (Figure S1). Of note, many of the frequently altered genes have been implicated in PCa, including HPN [35], CLDN8 (an androgen regulated gene that promotes PCa cell proliferation and migration) [36], and ONECUT2 (a known master regulator in PCa that suppresses the androgen axis) [37]. Hence, analysis of differentially expressed genes in the UTA and EMC cohorts highlighted candidate genes associated with PCa.

\subsection{Gene Graph Enrichment Analysis}

Data from transcription factor associated GRN identified in the preclinical prostate orthografts and individual gene sets from differentially expressed gene analysis on a per sample basis were integrated in a gene graph enrichment analysis (GGEA) to determine the activity status of the regulons (transcriptional regulators and their respective target genes) in the clinical tumours. The concordance of the positive and negative 'transcription factor-target gene' relationships was calculated for each sample within the UTA and EMC patient cohorts. GGEA [38] applies an enrichment approach to study the interactome surrounding the coregulators of interest to find supporting evidence of transcription factor activity. Differentially expressed genes in individual tumours within the two cohorts were mapped onto the candidate GRNs highlighted in the orthograft models.

To corroborate enriched gene networks shared among independent cohorts, we ranked the regulons by the respective frequency of activation in the UTA and EMC patient datasets (Figure S2). Consistently, among the ten most frequently active transcription factors (regulators) in these two datasets, we found three known genes implicated in PCa progression: $B A C H 1$ promotes invasion and migration of $\mathrm{PCa}$ cells by altering metastasis related genes [39]; CITED2 (Cbp/P300 Interacting Transactivator With Glu/Asp Rich Carboxy-Terminal Domain 2) has recently been proposed as a therapeutic target to tackle 
PCa metastasis [40]; and DNMT1 promotes PCa metastasis through the regulation of epithelial-mesenchymal transition and cancer stem cells [41]. Collectively, regulatory patterns identified in our preclinical orthograft PCa models successfully highlighted genes of potential clinical relevance.

\subsection{Prognostic Utility of Regulon Activity Status in Radical Prostatectomy Clinical Cohorts}

To evaluate the prognostic utility of the inferred regulons, we investigated the potential association between the enriched/not enriched status of regulons and the time to cancer relapse (signified by biochemical recurrence) following radical prostatectomy. We performed univariate CoxPH regression analysis in the UTA dataset in the first instance to identify enriched regulons associated with cancer recurrence (Table S4). Eleven statistically significant candidate regulons highlighted, with JMJD6 as the top-ranking enriched regulon ( $p=0.002$; Table 2A, Figure 2B, Table S5). Analysing the EMC cohort as a validation dataset, fourteen enriched regulons were identified. Consistent with findings from the UTA cohort, JMJD6 was also identified as the top-ranking enriched regulon ( $p=0.003$; Table 2B, Figure 2B, Table S5). Besides JMJD6, the SUFU regulon was enriched in both UTA and EMC cohorts. Analysing all available prostate cancer datasets in the cBio-portal $(n=22)$, altered JMJD6 gene was detected in multiple cohorts, with the highest incidence of genetic abnormalities (up to 8\%) detected in metastatic tumours (Figure S3). We reasoned that analysis of the JMJD6 regulon as a network, rather than at a single gene level, would provide additional insight into its functional impact. Univariate regression analysis further revealed that the active JMJD6 regulon was associated with early biochemical recurrence in both UTA (discovery) and EMC cohorts (Table 3A). We further examined the status of the JMJD6 regulon as a prognostic signature in the ICGC cohort for additional independent validation. Enrichment of the JMJD6 regulon significantly correlated with time to biochemical recurrence in the ICGC cohort in univariate analysis $(p=0.00648)$. Kaplan-Meier analysis for biochemical free survival further confirmed reduced biochemical free survival in the presence of active status for the JMJD6 regulon in patients within the UTA, EMC and ICGC cohorts (Figure 3).

Table 2. Univariate cox regression analysis for regulons enrichment. Top ten genes are listed for the (A) UTA and (B) EMC cohorts.

(A)

\begin{tabular}{ccc}
\hline Ensemb1 ID & Hugo Symbol & $p$ Value \\
\hline ENSG00000070495 & $J M J D 6$ & 0.002 \\
\hline ENSG00000196132 & MYT1 & 0.006 \\
\hline ENSG00000100410 & PHF5A & 0.02 \\
\hline ENSG00000065057 & NTHL1 & 0.02 \\
\hline ENSG00000159210 & SNF8 & 0.02 \\
\hline ENSG00000171222 & SCAND1 & 0.02 \\
\hline ENSG00000123091 & RNF11 & 0.02 \\
\hline ENSG00000120798 & NR2C1 & 0.02 \\
\hline ENSG00000107882 & $S U F U$ & 0.03 \\
\hline ENSG00000146083 & RNF44 & 0.04 \\
\hline Ensemb1 ID & $(\mathbf{B})$ & $p$ Value \\
\hline ENSG00000070495 & Hugo Symbol & 0.003 \\
\hline ENSG00000095002 & $J M J D 6$ & 0.006 \\
\hline ENSG00000107882 & $M S H 2$ & 0.007 \\
\hline
\end{tabular}


Table 2. Cont.

\begin{tabular}{ccc}
\hline ENSG00000136826 & KLF4 & 0.01 \\
\hline ENSG00000119969 & HELLS & 0.01 \\
\hline ENSG00000151929 & BAG3 & 0.02 \\
\hline ENSG00000105607 & GCDH & 0.02 \\
\hline ENSG00000092607 & TBX15 & 0.02 \\
\hline ENSG00000188486 & H2AFX & 0.02 \\
\hline ENSG00000180596 & HIST1H2BC & 0.03 \\
\hline
\end{tabular}

UTA

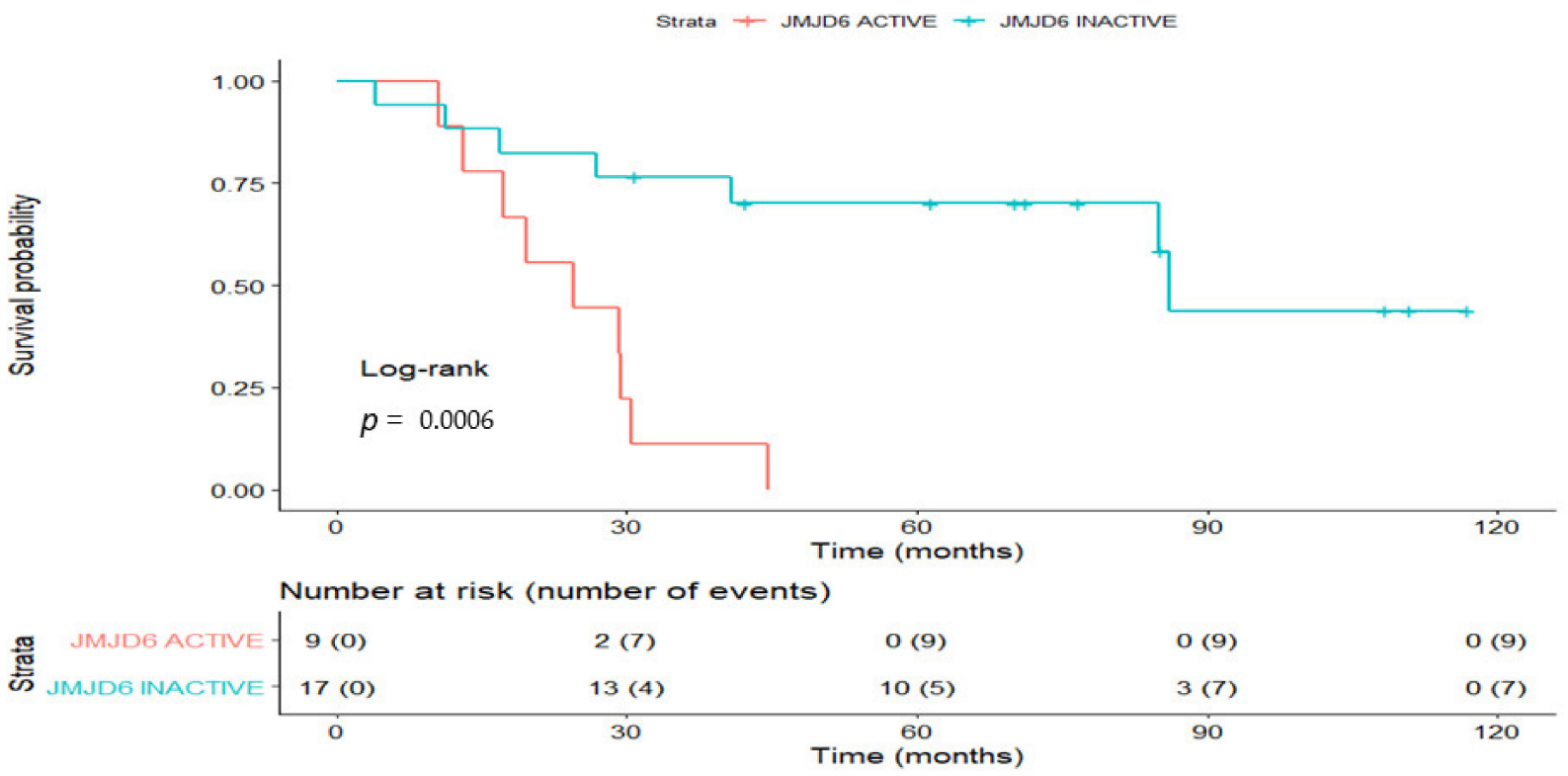

(A)

EMC

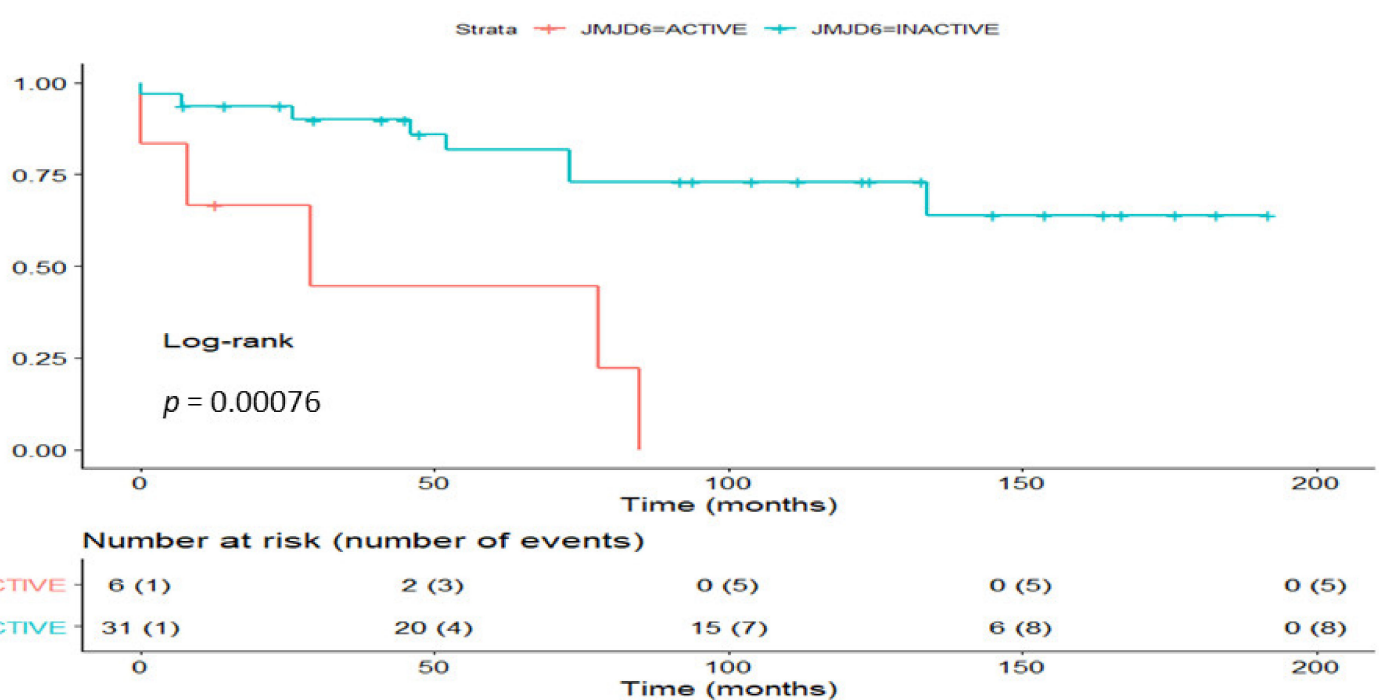

(B)

Figure 3. Cont. 


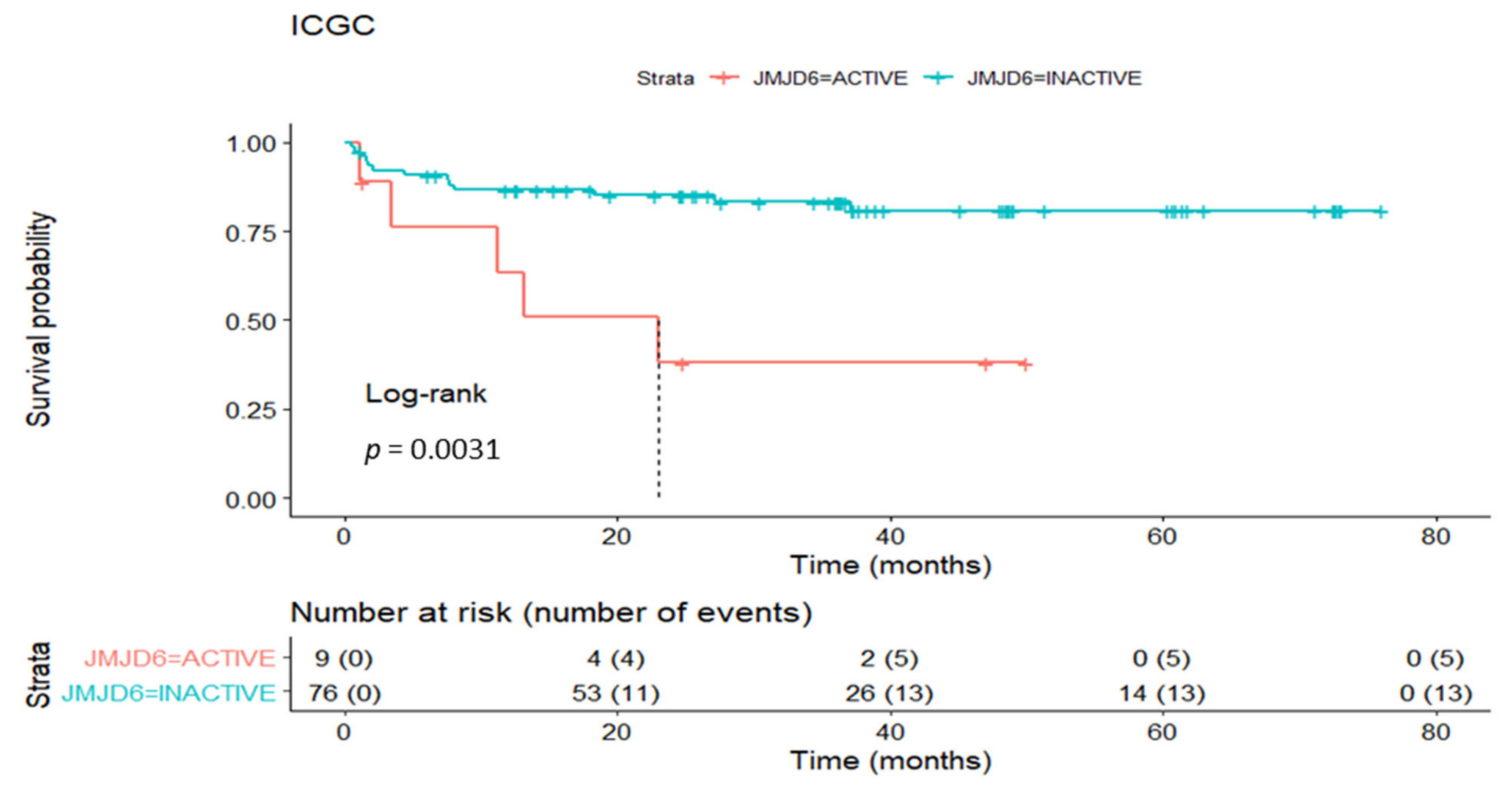

(C)

Figure 3. Disease free survival analysis of the JMJD6 regulon signature in clinical prostatectomy patient cohorts. The survival probability curves for patients in the UTA (A), EMC (B) and IGCG (C) cohorts were prepared with patients stratified according to the presence or absence of the enriched JMJD6 regulon in red and turquoise, respectively.

To benchmark the JMJD6 regulon as a prognostic marker for progressive/recurrent $\mathrm{PCa}$, three reported independent signatures were selected for comparison: two androgen receptor related signatures (namely TMEFF2 regulated cell cycle related gene signature [11] and the bromodomain related 10-genes signature [12]) as well as a 28-gene hypoxia signature [13]. The three signatures are referred to as TMCC11, BROMO-10 and HYPOXIA-28 respectively hereafter. Compared to JMJD6 being prognostic in all three cohorts, TMCC11 was prognostic in the UTA and ICGC cohorts but not the EMC cohort, while BROMO-10 and HYPOXIA-28 significantly predicted recurrence in only one of the three cohorts, UTA and ICGC, respectively (Table 3A). Multivariate analyses of the three signatures (and of the $J M J D 6$ regulon status) were performed if the respective univariate analysis were significant. In multivariate analysis, the JMJD6 regulon status significantly predicted disease recurrence in UTA and EMC, but not ICGC (Table 3B). Among the three published signatures, none significantly prognosticate for cancer recurrence in multivariate analysis.

Collectively, our analysis highlights the feasibility of integrating data from preclinical human orthograft models of PCa with multiple clinical cohorts to generate information on the regulon landscape in identifying potential prognostic signatures. For the first time, our data identified the active status of the JMJD6 regulon in patients at risk of PCa recurrence. 
Table 3. Cox regression univariate (A) and multivariate (B) survival analysis. $p$-values (P), Hazard ratios (HR) and $95 \%$ Confidence intervals (CI) are showed for each univariate regression. Multivariate analysis results, using Gleason score and/or tumour stage as covariates, are shown only for the variables whose association with biochemical recurrence was significant $(p<0.05)$ at univariate level. All significant $p$-values are highlighted in bold.

\begin{tabular}{|c|c|c|c|c|c|c|c|c|c|}
\hline \multicolumn{10}{|c|}{ (A) Univariate Analysis } \\
\hline \multirow{2}{*}{$\begin{array}{l}\text { Clinical } \\
\text { Cohorts } \\
\text { Statistics } \\
\end{array}$} & \multicolumn{3}{|c|}{ UTA } & \multicolumn{3}{|c|}{ EMC } & \multicolumn{3}{|c|}{ ICGC } \\
\hline & HR & $95 \% \mathrm{CI}$ & $p$ & HR & $95 \% \mathrm{CI}$ & $p$ & HR & $95 \% \mathrm{CI}$ & $p$ \\
\hline \multicolumn{10}{|c|}{ Clinicopathological variables } \\
\hline Gleason score & 2.7 & $1.6-4.7$ & 0.0004 & 1.9 & $0.2-16$ & 0.5 & 2 & $1.4-3$ & 0.0004 \\
\hline Tumor stage & 1.7 & $1-2.96$ & 0.05 & 1.3 & $1-1.6$ & 0.02 & 2.5 & $1.7-3.8$ & $<0.0001$ \\
\hline \multicolumn{10}{|c|}{ Signatures } \\
\hline active JMJD6 regulon & 6 & $1.9-18$ & 0.002 & 5.8 & $1.8-18.6$ & 0.003 & 4.2 & $1.5-12$ & 0.006 \\
\hline TMCC11 & 4.5 & $1.1-17.8$ & 0.03 & 1 & $0.3-3.7$ & 1.0 & 4 & $1.6-10.5$ & 0.004 \\
\hline BROMO-10 & 0.06 & $0.0069-0.52$ & 0.01 & 1.2 & $0.3-4.2$ & 0.8 & 2.6 & $0.7-9.3$ & 0.2 \\
\hline HYPOXIA-28 & 2.1 & $0.7-6.24$ & 0.2 & 1.1 & $0.4-3.5$ & 0.8 & 3.4 & $1.3-9.2$ & 0.01 \\
\hline \multicolumn{10}{|c|}{ (B) Multivariate analysis } \\
\hline & \multicolumn{3}{|c|}{ UTA } & \multicolumn{3}{|c|}{ EMC } & \multicolumn{3}{|c|}{ ICGC } \\
\hline & HR & $95 \% \mathrm{CI}$ & $\mathrm{P}$ & HR & $95 \% \mathrm{CI}$ & $\mathrm{P}$ & HR & $95 \% \mathrm{CI}$ & $\mathrm{P}$ \\
\hline JMJD6 regulon & 6.5 & $1.3-32$ & 0.02 & 4.4 & $1.3-14.6$ & 0.01 & 1.2 & $0.3-4.8$ & 0.7 \\
\hline Gleason score & 1.6 & $0.8-3.1$ & 0.2 & & & & 1.2 & $0.6-2.4$ & 0.6 \\
\hline Tumor stage & 2.3 & $1.1-4.9$ & 0.03 & 1.2 & $1-1.5$ & 0.05 & 2.6 & $1.3-4.9$ & 0.004 \\
\hline TMCC11 & 3.4 & $0.8-14.4$ & 0.1 & & & & 1.8 & $0.6-5.6$ & 0.3 \\
\hline Gleason score & 2.5 & $1.4-4.4$ & 0.002 & & & & 1.3 & $0.7-2.4$ & 0.5 \\
\hline Tumor stage & 1.58 & $0.8-3.2$ & 0.2 & & & & 2.2 & $1.1-4.4$ & 0.02 \\
\hline BROMO-10 & 0.3 & $0.03-4.2$ & 0.4 & & & & & & \\
\hline Gleason score & 2.15 & $1.08-4.27$ & 0.03 & & & & & & \\
\hline Tumor stage & 1.5 & $0.8-2.8$ & 0.2 & & & & & & \\
\hline HYPOXIA-28 & & & & & & & 2.1 & $0.7-6.1$ & 0.2 \\
\hline Gleason score & & & & & & & 1.3 & $0.7-2.4$ & 0.4 \\
\hline Tumor stage & & & & & & & 2.2 & $1.13-4.24$ & 0.02 \\
\hline
\end{tabular}

\section{Discussion}

We hypothesised that the study of genes positively and negatively regulated by one or more transcription factors (collectively referred to as regulons) is a suitable approach to capture the general mechanisms driving tumour progression in PCa [42]. For the first time, we integrated datasets from preclinical human prostate orthografts and clinical cohorts to investigate if specific regulons were associated with the outcome of patients with PCa. By mapping transcriptomic gene graph enrichment-based signatures on to a network of interacting gene regulators, we identify the JMJD6 regulon as a candidate prognostic signature for biochemical recurrent PCa. Our data is consistent with a recent report on GRN-based investigation in breast cancer [43]. Our data on JMJD6 in PCa is consistent with involvement of JMJD6 in oral [44], breast [45], neuroblastomas [46], melanoma [47] and ovarian [48] cancers.

The JMJD6 regulon consists of 27 positive and 3 negative putative target genes (Table S5), including RAD51, EZH2 and SORL1. RAD51 is predicted to be upregulated by JMJD6 (Figure 2B). RAD51, a critical gene for the DNA repair process, is upregulated in aggressive PCa [49], and is included as part of the panel in the U.S. Food and Drug Administration approved Prolaris gene expression assay [50]. Similarly, EZH2 (Enhancer of zeste homolog 2) is associated with PCa progression [51], and predicted to be upregulated 
by JMJD6 (Table S5). Lastly, the expression of SORL1, a known hypoxia regulated gene [29], negatively correlates with JMJD6 expression.

We successfully identified regulons of interest from preclinical prostate orthografts and then investigated the prognostic value of our top candidate JMJD6 regulon. Given the small number of preclinical samples available as a starting point to infer the GRNs in PCa, we were not able to robustly compare between hormone naïve and castration resistant orthografts. Instead, we combined the available orthografts to model tumour heterogeneity of clinical PCa. Importantly, some transcription factor-target genes relationships may not be revealed because of the limited sample number, thus creating potential biases with a subset of regulons appearing transcriptionally more important. Nonetheless, even with this limitation, the JMJD6 regulon was identified as a key regulon enriched in two independent clinical cohorts, namely UTA and EMC, as well as the published independent ICGC clinical cohort. The ICGC cohort consists of relatively young patients (mean: 47, range: $35-52$ years), compared to UTA (mean: 60 , range: $47-71$ years); such case selection bias may create confounding factors that contribute to the negative multivariate analysis for the JMJD6 regulon in the ICGC cohort.

Although androgen receptor (AR) is essential for both prostate organogenesis and carcinogenesis, to our surprise, AR was not identified as an enriched regulon in our analysis. AR may be functionally important in both benign and malignant prostatic epithelium, with distinct transcriptional profiles arising from functional re-programming. Even in CRPC, AR remains activated through by-pass mechanisms despite suppressed canonical (classical) androgen receptor pathway activities [52]. In addition, changes due to reprogramming of the AR as a transcription factor may not be fully highlighted by analysis of regulons as fixed transcription factor-target genes 'units'. Furthermore, AR splice variants (including AR-V7) are strongly implicated in CRPC. During the preparation of this report, a highly relevant publication highlighted the relationship between catalytic function of JMJD6 and the generation of AR-V7 mRNA in advanced prostate cancer [53]. Silencing of JMJD6 expression suppressed growth of LNCaP95 and 22Rv1 human CRPC cells, while combined JMJD6 knockdown and anti-androgen treatment with enzalutamide produced substantially more anti-proliferative effects than each of the two treatment alone. Collectively, their data implicates JMJD6 to be important in PCa cell viability and proliferation, thus further supporting our GRN-based findings.

The strategy of standardising the analysis, by adopting a panel of benign controls within each dataset (benign prostatic hyperplasia for the UTA and ICGC cohorts; benign tissue adjacent to the tumour for EMC cohort), allowed for the reduction of biases arising from different protocols for sample handling, sequencing and data processing. Indeed, by leveraging a panel of control samples within each cohort, it was possible to show commonalities among the independent data sets without resorting to batch correction.

JMJD6 belongs to the Jumonji C (JMJC) domain-containing family of proteins. JMJD6 is thought to function mainly as a lysyl 5-hydroxylase, and not as a demethylase [54], although enzymatically it has been shown to possess both catalytic activities. Its ability to regulate the transcriptional activity of p53 through hydroxylation of a lysine in the p53 C-terminus is highly relevant in cancer biology. Upregulated JMJD6 expression is related to tumour growth, tumour metastasis and high tumour pathological classification [55-57]. To build on our findings, the classical Waddington epigenetic landscape [58] model can be applied to describe in more detail the mechanism of regulation for the target genes within the JMJD6 regulon. Given its potential role in a number of tumour types, a novel JMJD6 specific inhibitor SKLB325 has recently been developed [48]. Should future research confirm JMJD6 as a driver gene for progressive PCa, formal evaluation of JMJD6 targeted therapy will be warranted. 


\section{Conclusions}

We have characterised gene regulatory networks from preclinical prostate orthografts and applied transcriptomic data from three clinical cohorts to identify the JMJD6 regulon as a potential prognostic marker in PCa.

Supplementary Materials: The following are available online at https:/ /www.mdpi.com/article/ 10.3390/cancers13092094/s1. Figure S1: Top ten differentially expressed genes (DEG) in the UTA and EMC clinical cohorts, Figure S2: Top 10 active regulons in the UTA and EMC clinical cohorts, Figure S3: Alteration frequency ( $Y$-axis) for JMJD6 gene across all 22 prostate cancer datasets in the c-Bio portal, Table S1: Starting list of transcription factors used for the analysis, Table S2: All regulons identified from preclinical models, Table S3: Network statistics per node, Table S4: Univariate Cox regression $p$-values from the analysis of the internal patient datasets, Table S5: JMJD6 regulon.

Author Contributions: Study design: M.C., M.G., Z.G., B.J., H.Y.L., I.J.B. Provision of data and subsequent analysis and interpretation: M.C., M.J.S., M.N., G.J., A.U., Z.G., G.H., H.Y.L., I.J.B.; Writing of manuscript: M.C., M.G., G.H., H.Y.L., I.J.B.; Critical review of manuscript: M.J.S., M.N., G.J., A.U., Z.G., B.J., G.H.; Obtain funding: B.J., H.Y.L. All authors have read and agreed to the published version of the manuscript.

Funding: This project and the TransPot Marie Skłodowska-Curie Innovative Training Network received funding from the European Union's Horizon 2020 research and innovation programme under the Marie Skłodowska-Curie grant agreement No 721746. M.C. is a Early Stage Researcher funded within the TransPot Marie Skłodowska-Curie Innovative Training Network. A.U. is supported by the Norwegian Cancer Cociety grant number \#198016-2018.

Institutional Review Board Statement: The study was conducted according to the guidelines of the Declaration of Helsinki. Specific approval by the Institutional Review Board is not applicable for our study was based on data previously published with relevant approval in place.

Informed Consent Statement: Not applicable.

Data Availability Statement: The data presented in this study are openly available. The orthograft sequencing data has been uploaded in array express at www.ebi.ac.uk/arrayexpress/ (accessed on 26 November 2020), with access details available from the corresponding authors. The codes used in the report are available at https://github.com/MarioCangiano/Cancers_article.git (accessed on 7 April 2021).

Acknowledgments: The authors thank the TransPot team for invaluable discussions and support for this project. We are especially grateful to Craig Robson and Linda Rushworth for their generous assistance, and to Catherine Winchester for review of the manuscript. The EMC dataset was obtained in collaboration with the NGS-ProToCol Consortium.

Conflicts of Interest: The authors declare no competing interests.
Abbreviations
PCa Prostate Cancer
GRN Gene regulatory network
AR Androgen Receptor

\section{References}

1. Siegel, R.L.; Miller, K.D.; Fuchs, H.E.; Jemal, A. Cancer Statistics 2021. CA Cancer J. Clin. 2021, 71, 7-33. [CrossRef]

2. Ciccarese, C.; Massari, F.; Iacovelli, R.; Fiorentino, M.; Montironi, R.; Di Nunno, V.; Giunchi, F.; Brunelli, M.; Tortora, G. Prostate cancer heterogeneity: Discovering novel molecular targets for therapy. Cancer Treat. Rev. 2017, 54, 68-73. [CrossRef]

3. Cornford, P.; Bellmunt, J.; Bolla, M.; Briers, E.; De Santis, M.; Gross, T.; Henry, A.M.; Joniau, S.; Lam, T.B.; Mason, M.D.; et al. EAU-ESTRO-SIOG Guidelines on Prostate Cancer. Part II: Treatment of Relapsing, Metastatic, and Castration-Resistant Prostate Cancer. Eur. Urol. 2017, 71, 630-642. [CrossRef]

4. Amaral, T.M.S.; Macedo, D.; Fernandes, I.; Costa, L. Castration-Resistant Prostate Cancer: Mechanisms, Targets, and Treatment. Prostate Cancer 2012, 2012, 1-11. [CrossRef] [PubMed]

5. Parimi, V.; Goyal, R.; Poropatich, K.; Yang, X.J. Neuroendocrine differentiation of prostate cancer: A review. Am. J. Clin. Exp. Urol. 2014, 2, 273-285. 
6. $\quad$ Myers, J.S.; Von Lersner, A.K.; Robbins, C.J.; Sang, Q.-X.A. Differentially Expressed Genes and Signature Pathways of Human Prostate Cancer. PLoS ONE 2015, 10, e0145322. [CrossRef] [PubMed]

7. Zeng, T.; Sun, S.-Y.; Wang, Y.; Zhu, H.; Chen, L. Network biomarkers reveal dysfunctional gene regulations during disease progression. FEBS J. 2013, 280, 5682-5695. [CrossRef]

8. Prolaris Cell Cycle Progression Test for Localized Prostate Cancer: A Health Technology Assessment. Ont. Health Technol. Assess. Ser. 2017, 17, 1-75.

9. Dalela, D.; Löppenberg, B.; Sood, A.; Sammon, J.; Abdollah, F. Contemporary Role of the Decipher®Test in Prostate Cancer Management: Current Practice and Future Perspectives. Rev. Urol. 2016, 18, 1-9.

10. Eeden, S.K.V.D.; Lu, R.; Zhang, N.; Quesenberry, C.P.; Shan, J.; Han, J.S.; Tsiatis, A.C.; Leimpeter, A.D.; Lawrence, H.J.; Febbo, P.G.; et al. A Biopsy-based 17-gene Genomic Prostate Score as a Predictor of Metastases and Prostate Cancer Death in Surgically Treated Men with Clinically Localized Disease. Eur. Urol. 2018, 73, 129-138. [CrossRef]

11. Dempster, J.; Krill-Burger, J.; Warren, A.; McFarland, J.; Golub, T.; Tsherniak, A. Gene expression has more power for predicting in vitro cancer cell vulnerabilities than genomics. bioRxiv 2020. [CrossRef]

12. Litwin, M.S.; Tan, H.J. The diagnosis and treatment of prostate cancer: A review. JAMA 2017, 317, 2532-2542. [CrossRef] [PubMed]

13. Creed, J.H.; Berglund, A.E.; Rounbehler, R.J.; Awasthi, S.; Cleveland, J.L.; Park, J.Y.; Yamoah, K.; Gerke, T.A. Commercial Gene Expression Tests for Prostate Cancer Prognosis Provide Paradoxical Estimates of Race-Specific Risk. Cancer Epidemiol. Biomark. Prev. 2020, 29, 246-253. [CrossRef] [PubMed]

14. Wang, Y.-H.; Wu, S.-Q.; Su, H.; Zhao, X.-K. Role of tumor-associated immune cells in prostate cancer: Angel or devil? Asian J. Androl. 2019, 21, 433-437. [CrossRef] [PubMed]

15. Krušlin, B.; Ulamec, M.; Tomas, D. Prostate cancer stroma: An important factor in cancer growth and progression. Bosn. J. Basic Med. Sci. 2015, 15, 1-8. [CrossRef]

16. Fitzgerald, K.A.; Evans, J.C.; McCarthy, J.; Guo, J.; Prencipe, M.; Kearney, M.; Watson, W.R.; O'Driscoll, C.M. The role of transcription factors in prostate cancer and potential for future RNA interference therapy. Expert Opin. Ther. Targets 2014, 18, 633-649. [CrossRef] [PubMed]

17. Grimes, T.; Potter, S.S.; Datta, S. Integrating gene regulatory pathways into differential network analysis of gene expression data. Sci. Rep. 2019, 9, 1-12. [CrossRef]

18. Mochida, K.; Koda, S.; Inoue, K.; Nishii, R. Statistical and Machine Learning Approaches to Predict Gene Regulatory Networks from Transcriptome Datasets. Front. Plant Sci. 2018, 9, 1770. [CrossRef]

19. Martinez, R.S.; Salji, L.M.; Ntala, R.C.; Blanco, G.B.R.; Hedley, A.; Clark, W.; Peixito, P.; Hervouet, E.; Renaude, E. Schlafen family member 5 (SLFN5) regulates LAT1-mediated mTOR activation in castration-resistant prostate cancer. bioRxiv 2020. [CrossRef]

20. Ylipää, A.; Kivinummi, K.; Kohvakka, A.; Annala, M.; Latonen, L.; Scaravilli, M.; Kartasalo, K.; Leppänen, S.-P.; Karakurt, S.; Seppälä, J.; et al. Transcriptome Sequencing Reveals PCAT5 as a Novel ERG-Regulated Long Noncoding RNA in Prostate Cancer. Cancer Res. 2015, 75, 4026-4031. [CrossRef]

21. Chen, S.; Huang, V.; Xu, X.; Livingstone, J.; Soares, F.; Jeon, J.; Zeng, Y.; Hua, J.T.; Petricca, J.; Guo, H.; et al. Widespread and Functional RNA Circularization in Localized Prostate Cancer. Cell 2019, 176, 831-843.e22. [CrossRef]

22. Hendriksen, P.J.; Dits, N.F.; Kokame, K.; Veldhoven, A.; Van Weerden, W.M.; Bangma, C.H.; Trapman, J.; Jenster, G. Evolution of the Androgen Receptor Pathway during Progression of Prostate Cancer. Cancer Res. 2006, 66, 5012-5020. [CrossRef] [PubMed]

23. Gerhauser, C.; Favero, F.; Risch, T.; Simon, R.; Feuerbach, L.; Assenov, Y.; Heckmann, D.; Sidiropoulos, N.; Waszak, S.M.; Hübschmann, D.; et al. Molecular Evolution of Early-Onset Prostate Cancer Identifies Molecular Risk Markers and Clinical Trajectories. Cancer Cell 2018, 34, 996-1011.e8. [CrossRef] [PubMed]

24. Fletcher, M.N.C.; Castro, M.A.A.; Wang, X.; De Santiago, I.; O’Reilly, M.; Chin, S.-F.; Rueda, O.M.; Caldas, C.; Ponder, B.A.J.; Markowetz, F.; et al. Master regulators of FGFR2 signalling and breast cancer risk. Nat. Commun. 2013, 4, 1-12. [CrossRef] [PubMed]

25. Liberzon, A.; Subramanian, A.; Pinchback, R.; Thorvaldsdóttir, H.; Tamayo, P.; Mesirov, J.P. Molecular signatures database (MSigDB) 3.0. Bioinformatics 2011, 27, 1739-1740. [CrossRef]

26. Alvarez, M.J.; Shen, M.J.A.Y.; Giorgi, F.M.; Lachmann, A.; Ding, B.B.; Ye, B.H.; Califano, A. Functional characterization of somatic mutations in cancer using network-based inference of protein activity. Nat. Genet. 2016, 48, 838-847. [CrossRef]

27. Geistlinger, L.; Csaba, G.; Zimmer, R. Bioconductor's EnrichmentBrowser: Seamless navigation through combined results of set-\& network-based enrichment analysis. BMC Bioinform. 2016, 17, 1-11.

28. Georgescu, C.; Corbin, J.M.; Thibivilliers, S.; Webb, Z.D.; Zhao, Y.D.; Koster, J.; Fung, K.-M.; Asch, A.S.; Wren, J.D.; Ruiz-Echevarría, M.J. A TMEFF2-regulated cell cycle derived gene signature is prognostic of recurrence risk in prostate cancer. BMC Cancer 2019, 19, 1-13. [CrossRef] [PubMed]

29. Yang, L.; Roberts, D.; Takhar, M.; Erho, N.; Bibby, B.A.; Thiruthaneeswaran, N.; Bhandari, V.; Cheng, W.-C.; Haider, S.; McCorry, A.M.; et al. Development and Validation of a 28-gene Hypoxia-related Prognostic Signature for Localized Prostate Cancer. EBioMedicine 2018, 31, 182-189. [CrossRef]

30. Urbanucci, A.; Barfeld, S.J.; Kytölä, V.; Itkonen, H.M.; Coleman, I.M.; Vodák, D.; Sjöblom, L.; Sheng, X.; Tolonen, T.; Minner, S.; et al. Androgen Receptor Deregulation Drives Bromodomain-Mediated Chromatin Alterations in Prostate Cancer. Cell Rep. 2017, 19, 2045-2059. [CrossRef] 
31. Hsu, C.L.; Liu, J.S.; Wu, P.L.; Guan, H.H.; Chen, Y.L.; Lin, A.C.; Ting, H.-J.; Pang, S.-T.; Yeh, S.-D.; Ma, W.-L.; et al. Identification of a new androgen receptor (AR) co-regulator BUD31 and related peptides to suppress wild-type and mutated AR-mediated prostate cancer growth via peptide screening and X-ray structure analysis. Mol. Oncol. 2014, 8, 1575-1587. [CrossRef]

32. Baek, J.-H.; Yun, H.S.; Kwon, G.T.; Lee, J.; Kim, J.-Y.; Jo, Y.; Cho, J.-M.; Lee, C.-W.; Song, J.-Y.; Ahn, J.; et al. PLOD3 suppression exerts an anti-tumor effect on human lung cancer cells by modulating the PKC-delta signaling pathway. Cell Death Dis. 2019, 10, 1-13. [CrossRef] [PubMed]

33. MSigDb. SCHAEFFER_PROSTATE_DEVELOPMENT_48HR_UP. Available online: https://www.gsea-msigdb.org/gsea/ msigdb/cards/SCHAEFFER_PROSTATE_DEVELOPMENT_48HR_UP (accessed on 1 August 2020).

34. Suyama, T.; Shiraishi, T.; Zeng, Y.; Yu, W.; Parekh, N.; Vessella, R.L.; Luo, J.; Getzenberg, R.H.; Kulkarni, P. Expression of cancer/testis antigens in prostate cancer is associated with disease progression. Prostate 2010, 70, 1778-1787. [CrossRef] [PubMed]

35. Goel, M.; Agrawal, D.; Natu, S.; Goel, A. Hepsin immunohistochemical expression in prostate cancer in relation to Gleason's grade and serum prostate specific antigen. Indian J. Pathol. Microbiol. 2011, 54, 476-481. [CrossRef]

36. Ashikari, D.; Takayama, K.-I.; Obinata, D.; Takahashi, S.; Inoue, S. CLDN8, an androgen-regulated gene, promotes prostate cancer cell proliferation and migration. Cancer Sci. 2017, 108, 1386-1393. [CrossRef]

37. Rotinen, M.; You, S.; Yang, J.; Coetzee, S.; Huang, W.-C.; Huang, F.; Pan, X.; Yyyez, A.; Hazelett, D.; Chu, C.-Y.; et al. ONECUT2 Is a Targetable Master Regulator of Lethal Prostate Cancer That Suppresses the Androgen Axis. SSRN Electron. J. 2018, $24,1887$. [CrossRef]

38. Geistlinger, L.; Csaba, G.; Küffner, R.; Mulder, N.; Zimmer, R. From sets to graphs: Towards a realistic enrichment analysis of transcriptomic systems. Bioinformatics 2011, 27, i366-i373. [CrossRef]

39. Shajari, N.; Davudian, S.; Kazemi, T.; Mansoori, B.; Salehi, S.; Shahgoli, V.K.; Shanehbandi, D.; Mohammadi, A.; Duijf, P.H.G.; Baradaran, B. Silencing of BACH1 inhibits invasion and migration of prostate cancer cells by altering metastasis-related gene expression. Artif. Cells Nanomed. Biotechnol. 2017, 46, 1495-1504. [CrossRef]

40. Shin, S.H.; Lee, G.Y.; Lee, M.; Kang, J.; Shin, H.W.; Chun, Y.S.; Park, J.W. Aberrant expression of CITED2 promotes prostate cancer metastasis by activating the nucleolin-AKT pathway. Nat. Commun. 2018, 9, 1-14. [CrossRef]

41. Lee, E.; Wang, J.; Yumoto, K.; Jung, Y.; Cackowski, F.C.; Decker, A.M.; Li, Y.; Franceschi, R.T.; Pienta, K.J.; Taichman, R.S. DNMT1 Regulates Epithelial-Mesenchymal Transition and Cancer Stem Cells, Which Promotes Prostate Cancer Metastasis. Neoplasia 2016, 18, 553-566. [CrossRef] [PubMed]

42. Garcia-Alonso, L.M.; Iorio, F.; Matchan, A.; Fonseca, N.A.; Jaaks, P.; Peat, G.; Pignatelli, M.; Falcone, F.; Benes, C.H.; Dunham, I.; et al. Transcription Factor Activities Enhance Markers of Drug Sensitivity in Cancer. Cancer Res. 2018, 78, 769-780. [CrossRef] [PubMed]

43. Castro, M.A.A.; De Santiago, I.; Campbell, T.M.; Vaughn, C.; Hickey, T.E.; Ross, E.; Tilley, W.D.; Markowetz, F.; Ponder, B.A.; Meyer, K.B. Regulators of genetic risk of breast cancer identified by integrative network analysis. Nat. Genet. 2016, 48, 12-21. [CrossRef]

44. Lee, C.-R.; Lee, S.H.; Rigas, N.K.; Kim, R.H.; Kang, M.K.; Park, N.-H.; Shin, K.-H. Elevated expression of JMJD6 is associated with oral carcinogenesis and maintains cancer stemness properties. Carcinogenesis 2015, 37, 119-128. [CrossRef]

45. Gao, W.-W.; Xiao, R.-Q.; Zhang, W.-J.; Hu, Y.-R.; Peng, B.-L.; Li, W.-J.; He, Y.-H.; Shen, H.-F.; Ding, J.-C.; Huang, Q.-X.; et al. JMJD6 Licenses ER $\alpha$-Dependent Enhancer and Coding Gene Activation by Modulating the Recruitment of the CARM1/MED12 Co-activator Complex. Mol. Cell 2018, 70, 340-357.e8. [CrossRef] [PubMed]

46. Wong, M.; Sun, Y.; Xi, Z.; Milazzo, G.; Poulos, R.C.; Bartenhagen, C.; Bell, J.L.; Mayoh, C.; Ho, N.; Tee, A.E.; et al. JMJD6 is a tumorigenic factor and therapeutic target in neuroblastoma. Nat. Commun. 2019, 10, 1-15. [CrossRef]

47. Liu, X.; Si, W.; Liu, X.; He, L.; Ren, J.; Yang, Z.; Yang, G.; Li, Y.; Liu, S.; Pei, F.; et al. JMJD6 promotes melanoma carcinogenesis through regulation of the alternative splicing of PAK1, a key MAPK signaling component. Mol. Cancer 2017, 16, 1-18. [CrossRef] [PubMed]

48. Zheng, H.; Tie, Y.; Fang, Z.; Wu, X.; Yi, T.; Huang, S.; Liang, X.; Qian, Y.; Wang, X.; Pi, R.; et al. Jumonji domain-containing 6 (JMJD6) identified as a potential therapeutic target in ovarian cancer. Signal Transduct. Target. Ther. 2019, 4, 1-14. [CrossRef]

49. Mitra, A.; Jameson, C.; Barbachano, Y.; Sanchez, L.; Kote-Jarai, Z.; Peock, S.; Sodha, N.; Bancroft, E.; Fletcher, A.; Cooper, C.; et al. Overexpression of RAD51 occurs in aggressive prostatic cancer. Histopathology 2009, 55, 696-704. [CrossRef]

50. Na, R.; Wu, Y.; Ding, Q.; Xu, J. Clinically available RNA profiling tests of prostate tumors: Utility and comparison. Asian J. Androl. 2016, 18, 575-579. [CrossRef]

51. Testa, U.; Castelli, G.; Pelosi, E. Cellular and Molecular Mechanisms Underlying Prostate Cancer Development: Therapeutic Implications. Medicines 2019, 6, 82. [CrossRef]

52. Mills, I.G. Maintaining and reprogramming genomic androgen receptor activity in prostate cancer. Nat. Rev. Cancer 2014, 14, 187-198. [CrossRef]

53. Paschalis, A.; Welti, J.; Neeb, A.J.; Yuan, W.; Figueiredo, I.; Pereira, R.; Ferreira, A.; Riisnaes, R.; Rodrigues, D.N.; Jiménez-Vacas, J.M.; et al. JMJD6 Is a Druggable Oxygenase That Regulates AR-V7 Expression in Prostate Cancer. Cancer Res. 2021, 81, 1087-1100. [CrossRef] [PubMed]

54. Unoki, M.; Masuda, A.; Dohmae, N.; Arita, K.; Yoshimatsu, M.; Iwai, Y.; Fukui, Y.; Ueda, K.; Hamamoto, R.; Shirakawa, M.; et al. Lysyl 5-Hydroxylation, a Novel Histone Modification, by Jumonji Domain Containing 6 (JMJD6)*. J. Biol. Chem. 2013, 288, 6053-6062. [CrossRef] [PubMed] 
55. Poulard, C.; Rambaud, J.; Lavergne, E.; Jacquemetton, J.; Renoir, J.-M.; Trédan, O.; Chabaud, S.; Treilleux, I.; Corbo, L.; Le Romancer, M. Role of JMJD6 in Breast Tumourigenesis. PLoS ONE 2015, 10, e0126181. [CrossRef] [PubMed]

56. Zhang, J.; Ni, S.-S.; Zhao, W.-L.; Dong, X.-C.; Wang, J.-L. High expression of JMJD6 predicts unfavorable survival in lung adenocarcinoma. Tumor Biol. 2013, 34, 2397-2401. [CrossRef]

57. Aprelikova, O.; Chen, K.; El Touny, L.H.; Brignatz-Guittard, C.; Han, J.; Qiu, T.; Yang, H.H.; Lee, M.P.; Zhu, M.; Green, J.E. The epigenetic modifier JMJD6 is amplified in mammary tumors and cooperates with c-Myc to enhance cellular transformation, tumor progression, and metastasis. Clin. Epigenetics 2016, 8, 1-16. [CrossRef] [PubMed]

58. Fard, A.T.; Ragan, M.A. Modeling the Attractor Landscape of Disease Progression: A Network-Based Approach. Front. Genet. 2017, 8, 48. [CrossRef] 